Revista Brasileira de Agricultura Irrigada v.12, nº.4, p. 2755 - 2760, 2018

ISSN 1982-7679 (On-line)

Fortaleza, CE, INOVAGRI - http://www.inovagri.org.br

DOI: $10.7127 /$ rbai.v12n400971

Protocolo 971.18 - 19/06/2018 Aprovado em 19/06/2018

\title{
ANÁLISE DO COMPORTAMENTO DE MODELOS PARA AJUSTE DA VELOCIDADE DE INFILTRAÇÃO EM DIFERENTES USOS DE SOLO
}

Raimundo Rodrigues Gomes Filho ${ }^{1}$, Igor Leonardo Nascimento Santos ${ }^{2}$, Alceu Pedrotti ${ }^{3}$; Clayton Moura de Carvalho ${ }^{4}$, Antenor Oliveira de Aguiar Netto ${ }^{5}$, Leonaria Luna Silva de Carvalho $^{6}$

\section{RESUMO}

Os testes foram realizados em uma área experimental da Universidade Federal de Sergipe (UFS), com o objetivo analisar o comportamento dos modelos matemáticos de Horton (1940), Kostiakov (1932), Kostiakov-Lewis (1945) e Phillip (1957) no ajuste da velocidade de infiltração em um ARGISSOLO VERMELHO AMARELO em diferentes manejos e com uso de diferentes culturas antecedentes. Os manejos de uso do solo consistiram de cultivo convencional, cultivo mínimo, plantio direto e cultivo de plantas em sucessão à cultura do milho variedade Biomatrix BM 3061. Foram utilizadas as plantas girassol (Helianthus annuus), milheto (Pennisetum glaucum (L.) R. Br.), guandu (Cajanus cajan) e crotalária (Crotalaria juncea) como culturas antecedentes. Em uma visão geral o modelo matemático de Philip obteve melhores resultados. Porém, dentro das limitações de cada modelo, todos geraram resultados estatisticamente equivalentes, exceto Kostiakov-Lewis.

Palavras-chave: plantio direto; Philip; Horton.

\section{ANALYSIS OF BEHAVIOR OF MODELS FOR ADJUSTING THE INFILTRATION RATE IN DIFFERENT SOIL USES.}

\footnotetext{
ABSTRACT

With the objective of analyze the behavior of the mathematical models of Horton (1940), Kostiakov (1932), Kostiakov-Lewis (1945), and Phillip (1957), the tests of water infiltration were realized in an experimental area of the Federal University of Sergipe (UFS) in a YELLOW RED ARGISSOL in different managements and using different antecedent crops. Soil use management consisted of conventional cultivation, minimum tillage, no-tillage and cultivation

${ }^{1}$ Doutor em Engenharia Agrícola, Professor do DEAGRI, UFS, Sergipe. rrgomesfilho@hotmail.com;

2 Engenheiro Agrícola, Mestrando em Recursos Hídricos, UFS, Sergipe. igorsantos1993@gmail.com;

${ }^{3}$ Doutor em Solos e Nutrição de plantas, Professor do DEA, UFS, Sergipe. alceupedrotti@gmail.com;

${ }^{4}$ Doutor em Engenharia Agrícola, Professor do IF Baiano, Serrinha, Bahia. clayton.carvalho@ifbaiano.edu.br;

${ }^{5}$ Doutor em Agronomia, Professor do DEA, UFS, Sergipe. Antenor.ufs@gmail.com;

${ }^{6}$ Mestra em Engenharia Agrícola, DENA/UFC, Fortaleza, Ceará. E-mail: leonarialuna@hotmail.com
} 
of antecedent crops in succession with the Biomatrix BM 3061 maize variety. The antecedent crops were sunflower (Helianthus annuus), millet (Pennisetum glaucum (L.) R. BR.), guandu (Cajanus cajan) and crotalaria (Crotalaria juncea). In an overview the mathematical model that on average obtained better results was Philip's. However, within the limitations of each model, all generated statistically equivalent results, with the exception of Kostiakov-Lewis.

Keywords: no-tillage; Philip; Horton.

\section{INTRODUÇÃO}

A infiltração de água no solo pode ser descrita como a passagem da água da superfície para o interior do solo, devido a morfologia encontrada no solo, a velocidade desse fenômeno é alta quando o solo está seco, por conta do grande número de macroporos vazios e tende a diminuir a medida que esses poros se preenchem com água, até que o solo se sature e velocidade se torne constante. Esse processo de saturação pode demorar horas em solos mais argilosos, por conta disso, testes de infiltração podem ser muito demorados e cansativos, levando pesquisadores a estudar metodologias e modelos matemáticos que facilitem a obtenção desse parâmetro físico do solo. Podem-se dividir os modelos matemáticos de infiltração de água no solo em duas categorias: empírico e determinísticos (HABILI; HEIDARPOUR, 2015).

As conclusões sobre o melhor modelo são diversas, Carvalho et al. (2015) chegaram a conclusão em seus experimentos que a metodologia proposta por Horton é a mais eficiente, já Cunha et al. (2015) concluíram que a metodologia de Kostiakov expressou melhor o processo de infiltração, mas Cunha et al. (2009) relataram em outro trabalho, também com diferentes modelos empíricos, que os modelos de Kostiakov e Horton foram semelhantes, do ponto de vista estatístico.

Portanto, este trabalho teve como objetivo analisar o comportamento dos modelos matemáticos de Horton (1940), Kostiakov (1932), Kostiakov-Lewis (1945) e Phillip (1957) no ajuste da velocidade de infiltração em um ARGISSOLO VERMELHO
AMARELO em diferentes manejos e com uso de diferentes culturas antecedentes.

\section{MATERIAL E MÉTODOS}

Os testes foram realizados na Estação Experimental Campus, da Universidade Federal de Sergipe (UFS), em um solo classificado como ARGISSOLO VERMELHO AMARELO, ou Ultisol para a USDA soil taxonomy.

O solo no qual o experimento foi realizado vem sendo manejado com três tipos de sistema de cultivo: cultivo convencional (composto de gradagem com grade niveladora de discos + aração com arado de discos + gradagem), cultivo mínimo (composto de 1 ou 2 gradagens com grade niveladora de discos, sendo que a segunda gradagem somente é realizada quando há incidência considerável de plantas espontâneas) e plantio direto (consistindo do não revolvimento do solo), também existe uma rotação de cultura, sendo cultura comercial milho variedade BM 3061 da Biomatrix, sucedido do cultivo de plantas de cobertura.

As plantas antecedentes ao cultivo comercial foram: girassol (Helianthus annuus), milheto (Pennisetum glaucum (L.) $R$. $B r$.$) , guandu (Cajanus cajan) e crotalária$ (Crotalaria juncea), representadas na Figura 1. Utilizou-se o esquema de faixas experimentais sendo os tratamentos de manejo de solo dispostos como faixas e os de culturas antecedentes como subparcelas com três repetições distribuídas ao acaso, totalizando 12 subparcelas em cada faixa. 

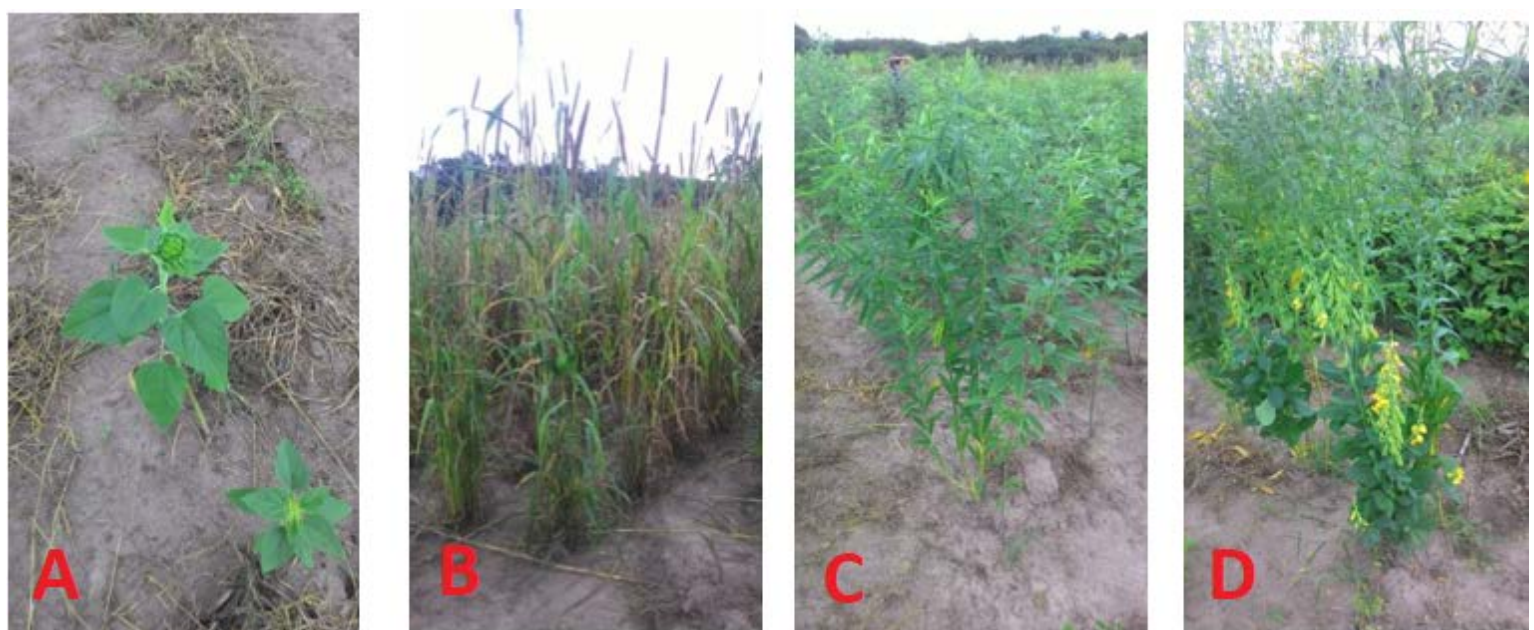

Figura 1. Parcelas com as plantas antecedentes girassol (A), milheto (B), guandu (C) e crotalária (D) utilizadas como cobertura do solo.

A obtenção dos dados da velocidade de infiltração foram realizadas logo após a colheita do milho, utilizando a metodologia dos duplos anéis infiltrômetros descrita por Brandão et al. (2006).

Para ajuste de equações foram utilizados os modelos empíricos propostos por Horton (1940), Kostiakov (1932), KostiakovLewis (1945) e Philip (1957), equações 1, 2, 3 e 4 respectivamente, os ajustes foram realizados utilizando o software VIBK (SANTOS et al., 2015).

$$
\begin{aligned}
& \mathrm{VI}=\mathrm{i}_{\mathrm{f}}+\left(\mathrm{i}_{\mathrm{i}}-\mathrm{i}_{\mathrm{f}}\right) e^{-\beta \mathrm{t}} \\
& \mathrm{VI}=\mathrm{k} \mathrm{t}^{\alpha} \\
& \mathrm{VI}=\mathrm{k} \mathrm{t}^{\alpha}+\mathrm{if}_{\mathrm{f}} \\
& \mathrm{VI}=\mathrm{f}_{1} \mathrm{t}^{-1 / 2}+\mathrm{f}_{2}
\end{aligned}
$$

Em que:

VI - velocidade de infiltração, mm.h ${ }^{-1}$; mm.h $\mathrm{h}^{-1}$

$i_{i}$ - velocidade de infiltração inicial,

if - velocidade de infiltração básica (VIB), mm.h ${ }^{-1}$;

$\mathrm{t}$ - tempo acumulado, $\mathrm{h}$;

$k, \alpha, \beta, \mathrm{f}_{1}$ e $\mathrm{f}_{2}$ - constantes obtidas empiricamente através de regressão.

Foram utilizados os índices estatísticos de coeficiente de massa residual (CMR), coeficiente de ajuste (CA), e eficiência (EF), equações 5, 6 e 7 respectivamente, para fazer a análise do desempenho dos modelos matemáticos, como descrito por Carvalho et al. (2015).

$$
\begin{gathered}
\mathrm{CMR}=\frac{\sum_{i=1}^{n} o_{i}-\sum_{i=1}^{n} P_{i}}{\left(\sum_{i=1}^{n} o_{i}\right)} \\
\mathrm{CA}=\frac{\sum_{i=1}^{n}\left(O_{i}-\bar{O}\right)^{2}}{\sum_{i=1}^{n}\left(P_{i}-\bar{O}\right)^{2}} \\
\mathrm{EF}=\frac{\sum_{i=1}^{n}\left(O_{i}-\bar{O}\right)^{2}-\sum_{i=1}^{n}\left(P_{i}-O_{i}\right)^{2}}{\sum_{i=1}^{n}\left(O_{i}-\bar{O}\right)^{2}}
\end{gathered}
$$

em que: $m m . h^{-1}$;

Oi - representa os valores observados,

$\mathrm{Pi}$ - representa os valores estimados, n é o número de observações, $\mathrm{mm} \cdot \mathrm{h}^{-1}$; $\mathrm{mm} \cdot \mathrm{h}^{-1}$;

$\bar{O}$ - a média aritmética das observações,

$\bar{P}$ - a média aritmética dos valores estimados, $\mathrm{mm} \cdot \mathrm{h}^{-1}$.

\section{RESULTADOS E DISCUSSÃO}

Na tabela 1 foram dispostos os resultados encontrados para todos os parâmetros estatísticos calculados. 
Tabela 1. Índices estatísticos das quatro culturas antecedentes nos três tipos de manejo

\begin{tabular}{ccccc}
\hline Modelos & $\mathrm{R}^{2}$ & CMR & CA & EF \\
\hline Observados & 1 & 0 & 1 & 1 \\
\hline Horton & 0,962 & 0,037 & 1,226 & 0,911 \\
\hline Kostiakov & 0,966 & 0,022 & 1,510 & 0,907 \\
\hline Kostiakov-Lewis & 0,988 & 0,152 & 4,476 & 0,562 \\
\hline Philip & 0,981 & 0 & 1,038 & 0,968 \\
\hline & \multicolumn{5}{c}{ Cultivo mínimo } \\
\hline Observados & 1 & 0 & 1 & 1 \\
\hline Horton & 0,921 & 0,082 & 1,272 & 0,815 \\
\hline Kostiakov & 0,923 & 0,035 & 1,907 & 0,812 \\
\hline Kostiakov-Lewis & 0,953 & 0,145 & 4,983 & 0,541 \\
\hline Philip & 0,947 & 0 & 1,119 & 0,898 \\
\hline \multicolumn{5}{c}{ Cultivo convencional } \\
\hline Observados & 1 & 0 & 1 & 1 \\
\hline Horton & 0,970 & 0,069 & 1,684 & 0,859 \\
\hline Kostiakov & 0,948 & 0,065 & 2,103 & 0,743 \\
\hline Kostiakov-Lewis & 0,985 & 0,296 & 5,662 & 0,324 \\
\hline Philip & 0,961 & 0 & 1,084 & 0,891 \\
\hline
\end{tabular}

$\mathrm{O}$ modelo que melhor ajustou a VIB em todos os tipos de manejos e usos de culturas antecedentes foi o de Horton, a VIB calculada foi igual a obtida no campo. Resultados semelhantes, onde o modelo de Hortan se ajustou melhor a VIB, foram encontrados por Carvalho et. al (2015), em um experimento com simulador de chuva em diferentes fases de desenvolvimento do milho e por Santos et. al (2014) em testes de infiltração no solo após o cultivo de feijão. Os modelos de Kostiakov e Philip tiveram uma tendência de subestimar as VIBs e o modelo de Kostiakov-Lewis uma tendência a superestimar. Em um trabalho realizado em um LATOSSOLO VERMELHO Distroférrico, Sobrinho et. al (2003) também encontraram resultados de VIB muito próximos aos encontrados em campos com o modelo de Horton, assim como uma grande superestimação no modelo de Kostiakov-Lewis. Já Cunha et. al (2015), realizando testes em um LATOSSOLO AMARELO, encontraram valores que subestimaram a VIB com o modelo de Kostiakov.
Em relação ao coeficiente de determinação $\left(\mathrm{R}^{2}\right)$, para todos os modelos em todos os tratamentos, os resultados foram satisfatórios, obtendo sempre um valor maior que $92 \%$ no coeficiente, em todos os manejos o método de Kostiakov-Lewis alcançou melhores resultados. Em média os melhores resultados foram encontrados no plantio direto e os piores no cultivo mínimo.

Houve um resultado bastante interessante no coeficiente de massa residual (CMR), onde valores positivos de CRM indicam que o modelo subestima as medidas de infiltração total e os valores negativos indicam que os superestima (CARVALHO, 2015), para o método de Philips, em todos os manejos ele se aproximou bastante do ideal, ou seja, a curva foi se ajustando muito bem, de maneira que praticamente não houve resíduos, também se pode afirmar que para esse modelo o volume de água infiltrada total calculado é a mesmo que o obtido em campo. Dentre os demais métodos o modelo de Kostiakov-Lewis obteve os resultados menos favoráveis. Para esse parâmetro estatístico o manejo de solo não se mostrou uniformemente influente, variando 
com o modelo matemático.

Sobre o coeficiente de ajuste (CA), os resultados foram satisfatoriamente semelhantes em todos os métodos, com exceção o de Kostiakov-Lewis, que não conseguiu se adequar ao solo. E mais uma vez o método de Philip obteve melhores resultados em todos os manejos.

Por último, foi estudada a eficiência dos modelos (EF), todos os testes, com exceção do de Kostiakov-Lewis, obtiveram bons resultados. Em relação ao manejo, houve uma tendência de piora no resultado a medida que a antropização do solo ficava mais forte.

Outra informação que pode ser retirada é a baixa eficiência em todos os parâmetros estatísticos do modelo de Kostiakov-Lewis em todos os manejos, ele só foi satisfatório no coeficiente de determinação $\left(\mathrm{R}^{2}\right)$, deixando evidente que apenas a análise do coeficiente de determinação não torna esse tipo de estudo estatístico confiável, além de mostrar que esse modelo tem problemas de subestimar o valor a partir de 10 minutos de experimento, fato já evidenciado por SOBRINHO et al. (2003), como também superestimar o valor da VIB.

\section{CONCLUSÕES}

O modelo matemático que obteve melhores resultados foi o de Philip. Porém, dentro das limitações de cada modelo, todos geraram resultados estatisticamente equivalentes, com exceção do de KostiakovLewis, que obteve resultados inferiores aos demais.

\section{REFERÊNCIAS}

BRANDÃO, V. dos S.; CECILIO, R. A.; PRUSKI, F. F.; SILVA, D. D. da. Infiltração da água no solo. 3. ed., atual. e ampl. Viçosa, MG: UFV, 2006. 120 p.

CARVALHO, D. F. de; EDUARDO, E. N.; ALMEIDA, W. S. de; SANTOS, L. A. F.; ALVES SOBRINHO, T. Water erosion and soil water infiltration in different stages of corn development and tillage systems. Revista Brasileira de Engenharia Agrícola e Ambiental, v. 19, n. 11, p. 10721078, 2015.

CUNHA, J. L. X. L.; ALBUQUERQUE, A. W.; SILVA, C. A.; ARAÚJO, E.; SANTOS, R. B. J. Velocidade de infiltração da água em um Latossolo Amarelo submetido ao sistema de manejo plantio direto. Caatinga, v. 22, n. 1, p. 199205, 2009.

CUNHA, J. L. X. L.; COELHO, M. E. H.; ALBUQUERQUE, A. W. de; SILVA, C. A.; SILVA JUNIOR, A. B. da; CARVALHO, I. D. de. Water infiltration rate in Yellow Latosol under different soil management systems. Revista Brasileira de Engenharia Agrícola e Ambiental, v. 19, n. 11, p. 10211027, 2015.

HABILI, J. M.; HEIDARPOUR, M. Application of the Green-Ampt model for infiltration into layered soils. Journal of Hydrology, v. 25, n. 527, p. 824832, 2015.

SANTOS, M. A. D. N. D., PANACHUKI, E., ALVES SOBRINHO, T., OLIVEIRA, P. T. S. D., \& RODRIGUES, D. B. B. Water infiltration in an ultisol after cultivation of common bean. Revista Brasileira de Ciência do Solo, v. 38, n. 5, p. 1612-1620, 2014.

SANTOS, I. L. N.; GOMES FILHO, R. R.; SANTOS, K. V.; MASSARANDUBA, W. de M.; CARVALHO, C. M. de. Software VIBK for estimated infiltration rate and water hydraulic conductivity in soil. In: Inovagri International Meeting, 3, 2015, Fortaleza. Anais... Fortaleza, INOVAGRI, 2015.

SOBRINHO, T. A.; VITORINO, A. C. T.; SOUZA, L. C. F. de; GONÇALVES, M. C.; CARVALHO, D. F. de. Infiltração de 
Gomes et al.

água no solo em sistemas de Brasileira de Engenharia Agrícola e plantio direto e convencional. Revista Ambiental, v. 7, n. 2, p. 191-196, 2003. 